\title{
El empoderamiento de las mujeres y la esperanza de vida al nacer en México
}

\author{
Álvaro J. Idrovo e Irene Casique ${ }^{2}$
}

Forma de citar

Idrovo AJ, Cacique I. El empoderamiento de las mujeres y la esperanza de vida la nacer en México. Rev Panam Salud Publica. 2006;20(1):29-38.

RESUMEN Objetivos. Evaluar el efecto del empoderamiento de las mujeres (EM) sobre la esperanza de vida al nacer (EVN) en los estados federativos de México y comparar los resultados de medir el EM con diferentes indicadores compuestos que privilegian en mayor o menor medida un enfoque individual o poblacional.

Métodos. Estudio ecológico con datos de los 32 estados federativos mexicanos. Se estimaron las correlaciones entre la EVN total y por sexo y la medida de empoderamiento de género (MEG), el indice de poder de decisión de la mujer en el hogar (IPDH), el indice de autonomía de la mujer (IAM), la desigualdad en el ingreso, algunos factores del ambiente físico, la proporción de la población que hablaba lengua indígena y la tasa migratoria neta. Mediante regresiones robustas se exploró el efecto de la MEG y los índices de autonomía y de poder de decisión de la mujer en el hogar sobre la EVN, ajustado por las demás variables independientes. Resultados. Se encontró una correlación inversa muy fuerte $(-0,93)$ entre la EVN total y el factor del ambiente físico que caracteriza la vulnerabilidad poblacional y la biodiversidad. También se encontraron correlaciones significativas, tanto directas como inversas, entre la EVN por una parte y el IPDH, el IAM, la tasa migratoria neta, el porcentaje de la población que hablaba lengua indígena y el coeficiente de Gini por la otra. Las regresiones robustas múltiples mostraron asociaciones inversas entre la MEG y la EVN en mujeres ( $\beta$ : $-1,44$; intervalo de confianza de 95\% [IC95\%]: -2,71 a-0,17). El IAM se asoció de manera directa con la EVN en hombres ( $\beta$ : 0,88; IC95\%: 0,01 a 1,75) y mujeres ( $\beta: 0,66 ;$ IC95\%: 0,03 a 1,30).

Conclusión. El uso de la MEG como aproximación al EM no puso de manifiesto efectos positivos del EM sobre la EVN en México. Se deben revisar los elementos que integran la MEG y la pertinencia de su uso en diversos contextos. El IAM mostró una mayor asociación con la EVN y su efecto fue de mayor magnitud en los hombres. Este indicador permitió medir la EM en México y se recomienda usarlo mientras no se tenga otro que permita captar más eficazmente todos los elementos que inciden en el EM.

Palabras clave Esperanza de vida, indicadores de salud, derechos de la mujer, México.

1 Centro de Investigación en Salud Poblacional, Instituto Nacional de Salud Pública, Cuernavaca, Morelos, México. La correspondencia se debe dirigir a Javier Idrovo, Centro de Investigación en Salud Poblacional, Instituto Nacional de Salud Pública, Av. Universidad 655, Colonia Santa María Ahuacatitlán, CP. 62508, Cuernavaca, Morelos, México. Correos electrónicos: idrovoaj@hotmail.com, idrovoaj @yahoo.com.mx
Se ha descrito la sociedad mexicana como un ejemplo típico de patriarcado, donde por largo tiempo se ha

\footnotetext{
2 Centro Regional de Investigaciones Multidisciplinarias (CRIM), Universidad Nacional Autónoma de México, Cuernavaca, Morelos, México.
}

aceptado la superioridad del hombre como parte del orden natural de las cosas (1) y las mujeres han carecido de poder en la esfera pública y privada (2). Sin embargo, en los últimos 50 años las mujeres mexicanas han logrado mayores niveles de educación y 
han participado más activamente en el mercado y en el sustento económico de la familia. Es así como la incorporación masiva de las mujeres al mercado laboral y los movimientos a favor de los derechos de la mujer y de la equidad de género han favorecido cambios en el ingreso familiar y en los procesos de toma de decisiones, lo que ha ayudado a que las mujeres tengan más poder dentro del hogar y de la sociedad en su conjunto (3).

La incorporación de las mujeres al mercado de trabajo no constituye por sí sola una vía de empoderamiento, pero es un elemento importante de ese proceso, más amplio, que también exige cambios en la ideología patriarcal prevaleciente. El empoderamiento de las mujeres (EM) puede definirse, entonces, como el proceso mediante el cual las mujeres logran superar su tradicional falta de acceso a los recursos $\mathrm{y}$ al poder y ganan paulatinamente un mayor control de las circunstancias que inciden en su vida. El EM abarca tanto un control sobre los recursos físicos, humanos, intelectuales y financieros, como un cambio en las creencias, los valores y las actitudes de la población. El EM, por tanto, es un proceso dirigido a cambiar la naturaleza y la dirección de las prácticas sociales que marginan a las mujeres $y$ puede beneficiar a otros sectores desfavorecidos de la sociedad (4), como el de los pobres, los homosexuales y los indígenas.

A pesar de los resultados científicos que apuntan a que el EM tiene efectos favorables sobre la salud, hay datos que demuestran lo contrario (5). La mayor educación materna suele asociarse con mejores niveles de salud infantil (6), aunque su efecto es diferente en los diversos estratos socioeconómicos (7); el EM favorece la participación de la mujer en los programas de promoción de la salud (8); el trabajo asalariado de las mujeres hace que sepan calificar mejor su estado de salud (9), especialmente en los niveles socioeconómicos más altos (10); y el mejoramiento del estatus de la mujer tiende a favorecer la disminución de la fertilidad (11) y el incremento de su esperanza de vida al nacer $(\mathrm{EVN})(12,13)$.
La mayoría de los estudios realizados han utilizado un enfoque individual y muy pocos han abordado, desde un enfoque poblacional, el efecto que tiene el EM sobre la salud (12-17).

Quizá el primer trabajo que abordó el tema de manera explícita fue el de Yllo, publicado en 1983 (15). En ese trabajo la autora construyó cuatro índices compuestos para medir el estatus de la mujer a nivel estatal en los Estados Unidos de América y estimó el efecto del EM sobre la violencia contra ella ocasionada por su pareja. Esos índices medían el nivel de autonomía económica, la educación, la participación política y el estatus legal de la mujer. Según esa investigación, en los estados donde había más violencia conyugal también se observó una mayor desigualdad de género. Este trabajo fue retomado por Kawachi et al. (14), quienes en estudios posteriores analizaron los efectos del EM con un enfoque epidemiológico explícito. El primer estudio utilizó un diseño ecológico basado en cuatro índices (participación política, autonomía económica, educación e ingresos y derechos reproductivos de las mujeres) para evaluar el efecto del estatus de la mujer sobre algunas medidas agregadas de morbilidad y mortalidad en los Estados Unidos. Según los resultados, se observó una asociación positiva entre cada uno de esos cuatro índices y la salud poblacional de hombres y mujeres, incluso después de ajustar según la desigualdad en el ingreso, las tasas de pobreza y la mediana del ingreso por hogar (14).

Más tarde, en un estudio con niveles múltiples de agregación, Kawachi et al. exploraron los efectos del estatus de la mujer - medida con los mismos cuatro indicadores - sobre el autoinforme de salud individual de 87848 mujeres. Los resultados demostraron que el estatus de la mujer tuvo un efecto independiente sobre el autoinforme de salud después de ajustar según factores macrodeterminantes conocidos (desigualdad en el ingreso y la mediana del ingreso estatal) y las características sociodemográficas individuales de las mujeres (16). Un estudio más reciente del mismo grupo analizó la relación de esos indicadores con los síntomas depresivos en 7789 mujeres y encontró un efecto contextual de la desigualdad por género sobre los síntomas depresivos, después de ajustar según la desigualdad en los ingresos y covariables sociodemográficas individuales de las mujeres estudiadas (17). Estos pocos estudios con enfoque poblacional han aportado pruebas cuantitativas - previamente descritas en estudios cualitativos - mediante indicadores que reflejan el estatus social, económico, político y educativo de la mujer, aunque no logran capturar la proyección multidimensional del EM.

Aunque ha habido valiosas excepciones $(2,3)$, en México los estudios sobre EM han seguido las tendencias internacionales $\mathrm{y}$ ha predominado el enfoque cualitativo e individual. Por lo tanto, no se ha explorado sistemáticamente el efecto del EM en niveles de agregación mayores (por ejemplo, en localidades, regiones o estados federativos) y no se han realizado mediciones que pudieran reflejar la multidimensionalidad del EM.

Ante la falta de investigaciones cuantitativas, de un enfoque poblacional explícito y de la exploración de diversas formas de medición del EM, se realizó este estudio con el objetivo de evaluar el efecto del EM sobre la EVN en los estados federativos de México y comparar los resultados de medir el EM con diferentes indicadores compuestos que privilegian en mayor o menor medida un enfoque individual o poblacional.

\section{MATERIALES Y MÉTODOS}

\section{Diseño y evento estudiado}

Se realizó un estudio ecológico analítico de grupos múltiples (18) con datos de los 32 estados federativos mexicanos. La EVN por sexo para cada uno de los estados para 2003 se obtuvo de los registros del Consejo Nacional de Población (CONAPO), que se encuentran recopilados por el Instituto Nacional de Estadística, Geografía e Informática (INEGI) (19). La EVN es 
una variable compuesta (18) que puede definirse como el número promedio de años adicionales que una persona podría vivir si las tendencias actuales en la mortalidad se mantuvieran iguales por el resto de su vida (20). La esperanza de vida depende en gran medida de los criterios usados para seleccionar los grupos. Por ejemplo, la EVN en regiones con elevada mortalidad infantil varía considerablemente según la mortalidad en los primeros años de vida, lo que no ocurre cuando se analiza la esperanza de vida a otras edades (21). Al utilizar la EVN se evitan problemas derivados de no tener en cuenta el lapso entre el momento en que ocurre la exposición y el momento en el que se presenta el evento. La EVN refleja la mortalidad infantil que más se asocia con las condiciones contemporáneas (22).

\section{La medición del empoderamiento de la mujer}

Para cumplir con los objetivos propuestos se aplicaron dos formas de medir el nivel de EM.

Mediciones multidimensionales con enfoque poblacional. Se utilizó la medida de empoderamiento de género (MEG) propuesta en 1995 por el Programa de las Naciones Unidas para el Desarrollo (PNUD) para medir el empoderamiento relativo de las mujeres y los hombres en las esferas de las actividades política y económica (23). La MEG se compone de tres variables que intentan reflejar las diversas dimensiones del EM y se apoya en un enfoque poblacional de la participación femenina en: a) la política, medida por la proporción de escaños parlamentarios ocupados por mujeres; b) las oportunidades profesionales, medidas por la proporción de puestos administrativos, ejecutivos, profesionales y técnicos ocupados por mujeres; y c) la posición económica, medida a través de los salarios pagados a las mujeres mediante el PIB per cápita sin ajuste. Con las tres variables descritas se determinaron los porcentajes equivalentes de igualdad en la distribución (PEID) para la participación parlamentaria, el empleo y el ingreso de las mujeres y se ponderan con igual peso. La participación femenina en cada esfera se calculó según la fórmula:

$$
\begin{gathered}
\text { PEID }=\left\{\left[(\text { índice femenino })^{1-\epsilon}\right]+\right. \\
\left.\left[(\text { índice masculino })^{1-\epsilon}\right]\right\}^{1 / 1-\epsilon}
\end{gathered}
$$

donde $\in$ mide la aversión a la desigualdad y se le asigna un valor de 2, ya que esa es la media armónica a la desigualdad, según la aproximación de Atkinson (24).

Los índices masculino y femenino quedaron determinados para cada uno de los tres ámbitos en los que se mide la desigualdad entre mujeres y hombres. Posteriormente, los tres PEID se promediaron para obtener la MEG:

$$
\begin{gathered}
\mathrm{MEG}=\left[\left(\mathrm{PEID}_{\text {representación parlamentaria }}\right)+\right. \\
\left.\left(\mathrm{PEID}_{\text {empleo }}\right)+\left(\text { PEID }_{\text {ingreso }}\right)\right] / 3
\end{gathered}
$$

Una explicación más detallada de la forma de construir este índice ya ha sido publicada (23). De esta manera, cuanto mayor sea la MEG, mayor será el EM en el estado federativo correspondiente. Los datos de cada una de los estados mexicanos para el año 2000 se tomaron del trabajo de María Eugenia Ibarrarán y Carlos Robles (25), quienes a su vez obtuvieron los datos del INEGI, la CONAPO, la Cámara de Senadores del Congreso de la Unión y el Programa Nacional de la Mujer (PRONAM).

Mediciones unidimensionales con enfoque individual. Se utilizaron dos índices compuestos: el de poder de decisión de la mujer en el hogar (IPDH) y el de la autonomía de la mujer (IAM). Ambos caracterizan dimensiones específicas del EM y se calcularon con los datos de la Encuesta Nacional sobre la Dinámica de las Relaciones en los Hogares 2003 (ENDIREH) (26) según una metodología ya establecida (27). Brevemente, el IPDH se basó en las respuestas dadas a 11 preguntas sobre quién toma la decisión en el hogar (calificadas como 0: solo el hombre; 1: ambos integrantes de la pareja; y 2: solo la mujer). Según el análisis factorial (28), con esas preguntas se pueden caracterizar muchas decisiones separadas en cuatro componentes: la crianza de los niños, los temas reproductivos, los gastos mayores y los gastos cotidianos, con porcentajes de la varianza de $28,1 \% ; 11,9 \% ; 9,3 \%$ y $8,1 \%$, respectivamente. Cada uno de esos componentes se estandarizó y se ponderó según el porcentaje de la varianza de cada uno (0,5 para el primer componente, 0,2 para el segundo, 0,16 para el tercero y 0,14 para el último). Una vez sumados se obtuvo un índice de 0 a 10, en el que los mayores puntajes correspondieron a un mayor poder de decisión de la mujer en el hogar.

El IAM se elaboró a partir de cuatro preguntas que medían la autonomía de la mujer para salir de compras, visitar a sus parientes, visitar a sus amistades y salir a divertirse, cuyas posibles respuestas fueron: "pide permiso", "avisa", "lo hace sin avisar o pedir permiso" y "no lo hace/no va sola/va con él" (27). La coherencia de estas preguntas fue evaluada mediante el coeficiente alfa de Cronbach (29). La puntación total podía estar entre 0 y 12 y el mayor valor correspondía a una mayor autonomía de la mujer.

En 11 de los 39 Estados (Baja California, Chiapas, Chihuahua, Coahuila, Hidalgo, Michoacán, Nuevo León, Quintana Roo, Sonora, Yucatán y Zacatecas) se establecieron convenios especiales con el INEGI para conformar muestras representativas de su población femenina (26). En el resto de los estados, aunque la muestra solo era representativa de las mujeres mayores de 15 años de edad que acudieron a los servicios de salud públicos de primer y segundo nivel, los valores promedio de cada índice se consideraron representativos de la población en esos estados, ya que este tipo de inferencia ha demostrado ser útil en otros estudios exploratorios en epidemiología social (30).

\section{Covariables}

En estudios previos se observó que los valores de la EVN en los diversos 
estados mexicanos están asociados con la desigualdad en el ingreso, el porcentaje de la población mayor de 5 años de edad que hablaba lengua indígena (31) y algunos factores del ambiente físico, como la vulnerabilidad social de la población y la biodiversidad (FC1), la urbanización, la industrialización y la sustentabilidad ambiental (FC2), la adaptabilidad ecológica (FC3) y los ambientes libres de plagas forestales (FC4). Los coeficientes de Gini utilizados para medir la desigualdad en el ingreso en el año 2000 se encuentran publicados en otro trabajo (32). A medida que aumenta la concentración de la riqueza, el valor de los coeficientes de Gini se acerca a la unidad, mientras que cuando la distribución del ingreso es más igualitaria, se acerca a cero (33). Los datos sobre la población que hablaba lenguas indígenas en 2000 se obtuvieron del INEGI (19). Los cuatro factores del ambiente físico se construyeron mediante análisis factorial exploratorio de 50 variables ambientales recopiladas por la Secretaría del Medio Ambiente y Recursos Naturales (SEMARNAT) (34); una descripción más detallada de la construcción de los factores ambientales se encuentra en otra publicación ${ }^{3}$.

Para controlar un posible sesgo en la selección (sesgo de migración) se ajustó la estimación del efecto del EM en función de la tasa neta de migración, según los datos del saldo neto migratorio anual (número de inmigrantes menos el número de emigrantes en un año) de la población mayor de 5 años de edad entre 1995 y 2000, obtenidos del INEGI (19). Todas las covariables mencionadas se utilizaron para ajustar el efecto del EM sobre la EVN.

\section{Análisis estadístico}

Inicialmente se exploró la correlación entre la EVN y los indicadores de EM, la desigualdad en el ingreso, el porcentaje de la población mayor de 5 años de

\footnotetext{
3 Idrovo AJ, Hernández-Ávila M. Impacto del ambiente físico sobre la esperanza de vida al nacer en México: un estudio eco-epidemiológico. (Enviado a Salud Pública de México para su publicación).
}

edad que hablaba lenguas indígenas, los factores del ambiente físico y la tasa migratoria neta, mediante la prueba de Spearman y sus respectivos intervalos de confianza de 95\% (IC95\%). Posteriormente se estimaron los efectos brutos de los indicadores de EM, las demás variables independientes y los términos de interacción entre la MEG, el IPDH y el IAM, y el coeficiente de Gini para la EVN - total y por sexo-, mediante regresiones robustas. En las regresiones múltiples solo se emplearon las variables independientes de EM, el coeficiente de Gini, los factores FC3 y FC4 y la tasa migratoria neta, que mostraron valores de $P<0,05$ en el análisis bifactorial y el término de interacción entre el coeficiente de Gini y el IPDH $(P<0,15)$.

El uso de regresiones robustas permitió obtener estimaciones precisas sin necesidad de cumplir los supuestos de la regresión lineal convencional. Con esta técnica se estima primero una regresión lineal y se calculan las distancias de Cook para cada observación, lo que permite determinar la influencia que tiene cada observación sobre los estimadores obtenidos. Posteriormente se eliminan del análisis las observaciones con una distancia de Cook $>1$ y para terminar se realiza una nueva estimación mediante iteraciones de Huber y biponderadas (35), de acuerdo con las distancias de Cook calculadas previamente. Se utilizó un nivel de significación $P<0,05$, excepto para los términos de interacción, donde se utilizó un valor de $P<0,15$. Todos los análisis se hicieron con el programa estadístico Stata 9 (Stata Corporation, College Station, Texas).

\section{RESULTADOS}

La EVN total para México en 2003 fue de 74,9 años, con una desviación estándar (DE) de 0,75, mayor para las mujeres (77,3 años; DE: 0,66) que para los hombres (72,4 años; DE: 0,85). La mediana nacional de la MEG para el año 2000 fue de 0,56, con valores extremos en Querétaro $(0,37)$ y Baja California Sur $(0,69)$. El valor promedio nacional del IPDH fue de 4,46 (DE: 0,24), con el valor mínimo en Chiapas $(3,80)$ y el máximo en Aguascalientes $(5,03)$. El promedio del IAM para México fue de 6,38 (DE: 0,34), con valores extremos en Chiapas $(0,34)$ y Tamaulipas $(7,07)$.

En el cuadro 1 se presentan las correlaciones entre la EVN y las variables independientes estudiadas. Se encontró una correlación inversa muy fuerte $(-0,93)$ entre la EVN total y el factor que expresa la vulnerabilidad/ susceptibilidad poblacional y la biodiversidad (FC1). También se encontraron correlaciones significativas, tanto directas como inversas, según el coeficiente de Spearman ( $\rho \geq \pm 0,6 ; P<$ $0,05)$ entre la EVN por una parte y el IPDH, el IAM, la tasa migratoria neta, el porcentaje de la población que hablaba lengua indígena y el coeficiente de Gini por el otro; entre el FC1 y el porcentaje de la población que hablaba lengua indígena; y entre el IPDH y el FC1. También se observaron correlaciones significativas $(\rho< \pm 0,6 ; P<0,05)$ entre el coeficiente de Gini y el IPDH y entre el IAM por una parte y el FC1 y el porcentaje de la población que hablaba lengua indígena; entre la MEG y el FC2; entre la tasa migratoria neta por una parte y la EVN, el IPDH, el IAM y el FC1 por la otra; entre el IPDH $y$ el porcentaje que hablaba lengua indígena; y entre el FC4 y el IAM. El alto grado de correlación entre las variables es un hecho encontrado frecuentemente en este tipo de estudios debido, entre otras causas, a que suelen tener factores determinantes en común (36).

En la figura 1 se pueden ver las relaciones encontradas entre la EVN total y la MEG, el IPDH y el IAM. En el primer caso no se observó un patrón claro, mientras que en los otros dos la relación fue directa y lineal. Estas y las relaciones brutas entre las demás variables independientes y la EVN según el sexo se presentan en el cuadro 2 . Nótese que las variables asociadas significativamente $(P<0,05)$ con la EVN total y por sexo son las mismas y que los valores del coeficiente de Gini presentaron una mayor fuerza. El efecto negativo evidenciado por el coeficiente de Gini resultó mayor para los hombres que para las mujeres, es 
CUADRO 1. Correlaciones de Spearman entre la esperanza de vida al nacer, el empoderamiento de la mujer, la desigualdad en el ingreso, el ambiente físico $\mathrm{y}$ la tasa migratoria neta, en los estados federativos mexicanos, 2003

\begin{tabular}{|c|c|c|c|c|c|c|c|c|c|c|}
\hline Variables & $\begin{array}{c}\text { Esperanza } \\
\text { de vida al } \\
\text { nacer, total }\end{array}$ & $\mathrm{MEG}^{\mathrm{C}}$ & $\begin{array}{l}\text { Índice de } \\
\text { poder de } \\
\text { decisión }{ }^{b}\end{array}$ & $\begin{array}{l}\text { Índice de } \\
\text { autonomía }\end{array}$ & $\begin{array}{l}\text { Coeficiente } \\
\text { de Gini }\end{array}$ & $\begin{array}{c}\text { Población } \\
\text { con lengua } \\
\text { indígena } \\
(\%)\end{array}$ & $\mathrm{FC} 1$ & FC2 & FC3 & FC4 \\
\hline $\mathrm{MEG}^{\mathrm{C}}$ & $\begin{array}{c}-0,13 \\
(-0,46 ; 0,23)\end{array}$ & 1 & & & & & & & & \\
\hline $\begin{array}{l}\text { Índice de } \\
\text { poder de } \\
\text { decisión en } \\
\text { el hogar }\end{array}$ & $\begin{array}{c}0,60^{d} \\
(0,31 ; 0,78)\end{array}$ & $\begin{array}{c}-0,03 \\
(-0,38 ; 0,32)\end{array}$ & 1 & & & & & & & \\
\hline $\begin{array}{l}\text { Índice de } \\
\text { autonomía } \\
\text { de la mujer }\end{array}$ & $\begin{array}{c}0,62^{\mathrm{d}} \\
(0,35 ; 0,80)\end{array}$ & $\begin{array}{c}-0,12 \\
(-0,45 ; 0,24)\end{array}$ & $\begin{array}{c}0,31 \\
(-0,04 ; 0,60)\end{array}$ & 1 & & & & & & \\
\hline $\begin{array}{l}\text { Coeficiente } \\
\text { de Gini }\end{array}$ & $\begin{array}{c}-0,60^{\mathrm{d}} \\
(-0,79 ; 0,32)\end{array}$ & $\begin{array}{c}-0,06 \\
(-0,40 ; 0,29)\end{array}$ & $\begin{array}{c}-0,42^{\mathrm{d}} \\
(-0,67 ;-0,08)\end{array}$ & $\begin{array}{c}-0,37^{\mathrm{d}} \\
(-0,64 ;-0,02)\end{array}$ & 1 & & & & & \\
\hline $\begin{array}{l}\text { Población } \\
\text { con lengua } \\
\text { indígena (\%) }\end{array}$ & $\begin{array}{c}-0,69^{\mathrm{d}} \\
(-0,84 ;-0,45)\end{array}$ & $\begin{array}{c}0,18 \\
(-0,18 ; 0,50)\end{array}$ & $\begin{array}{c}-0,48^{d} \\
(-0,71 ;-0,16)\end{array}$ & $\begin{array}{c}-0,28 \\
(-0,57 ; 0,08)\end{array}$ & $\begin{array}{c}0,39^{d} \\
(0,05 ; 0,65)\end{array}$ & 1 & & & & \\
\hline FC3 & $\begin{array}{c}0,08 \\
(-0,27 ; 0,42)\end{array}$ & $\begin{array}{c}-0,12 \\
(-0,45 ; 0,24)\end{array}$ & $\begin{array}{c}-0,02 \\
(-0,37 ; 0,33)\end{array}$ & $\begin{array}{c}0,04 \\
(-0,32 ; 0,38)\end{array}$ & $\begin{array}{c}0,14 \\
(-0,22 ; 0,47)\end{array}$ & $\begin{array}{c}-0,03 \\
(-0,37 ; 0,32)\end{array}$ & $\begin{array}{c}0,02 \\
(-0,33 ; 0,37)\end{array}$ & $\begin{array}{c}0,24 \\
(-0,12 ; 0,54)\end{array}$ & 1 & \\
\hline FC4 & $\begin{array}{c}0,15 \\
(-0,21 ; 0,48)\end{array}$ & $\begin{array}{c}0,06 \\
(-0,29 ; 0,40)\end{array}$ & $\begin{array}{c}0,07 \\
(-0,28 ; 0,41)\end{array}$ & $\begin{array}{c}0,36^{d} \\
(0,02 ; 0,63)\end{array}$ & $\begin{array}{c}-0,28 \\
(-0,57 ; 0,08)\end{array}$ & $\begin{array}{c}0,13 \\
(-0,23 ; 0,46)\end{array}$ & $\begin{array}{c}-0,06 \\
(-0,40 ; 0,30)\end{array}$ & $\begin{array}{c}-0,02 \\
(-0,36 ; 0,33)\end{array}$ & $\begin{array}{c}0,19 \\
(-0,17 ; 0,51)\end{array}$ & 1 \\
\hline $\begin{array}{l}\text { Tasa migratoria } \\
\text { neta }\end{array}$ & $\begin{array}{c}0,61^{\mathrm{d}} \\
(0,33 ; 0,79)\end{array}$ & $\begin{array}{c}-0,13 \\
(-0,46 ; 0,23)\end{array}$ & $\begin{array}{c}0,42^{\mathrm{d}} \\
(0,08 ; 0,67)\end{array}$ & $\begin{array}{c}0,43^{\mathrm{d}} \\
(0,09 ; 0,67)\end{array}$ & $\begin{array}{c}-0,31 \\
(-0,59 ; 0,05)\end{array}$ & $\begin{array}{c}-0,31 \\
(-0,59 ; 0,04)\end{array}$ & $\begin{array}{c}-0,57^{d} \\
(-0,77 ;-0,28)\end{array}$ & $\begin{array}{c}-0,26 \\
(-0,56 ; 0,10)\end{array}$ & $\begin{array}{c}-0,12 \\
(-0,45 ; 0,24)\end{array}$ & $\begin{array}{c}0,23 \\
(-0,13 ; 0,53)\end{array}$ \\
\hline
\end{tabular}

Fuente: Estimado con datos de la Encuesta Nacional sobre la Dinámica de las Relaciones en los Hogares 2003 (ENDIREH) (26).

${ }^{a}$ FC1: vulnerabilidad/susceptibilidad poblacional y biodiversidad; FC2: urbanización, industrialización y sustentabilidad ambiental; FC3: resiliencia ecológica; FC4: ambientes libres de plagas forestales.

${ }^{b}$ Los intervalos de confianza del $95 \%$ se encuentran entre paréntesis.

${ }^{c}$ MEG: Medida de empoderamiento de género, propuesta por el Programa de las Naciones Unidas para el Desarrollo.

${ }^{d}$ Diferencia significativa $(P<0,05)$.

decir, al aumentar la desigualdad en el ingreso, la EVN disminuyó más en los hombres que en las mujeres.

Las otras variables asociadas con la EVN fueron el IPDH, el IAM, el FC1, la tasa migratoria neta y el hablar una lengua indígena. Las asociaciones de los dos índices y de la tasa migratoria neta fueron directas, por lo que al aumentar las dimensiones del EM medidas con esos índices aumentó la EVN y cuando la emigración era mayor que la inmigración también aumentó la EVN. Sin embargo, las otras variables mostraron asociaciones inversas, es decir, al aumentar la vulnerabilidad o susceptibilidad poblacional, la biodiversidad y la proporción de la población que hablaba lengua indígena, disminuyó la EVN. Resulta llamativo el hecho de que el efecto de las variables independientes resultó mayor entre los hombres que entre las mujeres. La interacción entre la MEG y el coeficiente de Gini mostró diferencias significativas en los hombres $(P=0,13)$ y marginalmente significativas $(P=0,17)$ en las mujeres, mientras que la interacción entre el coeficiente de Gini y el IPDH fue significativa para las mujeres $(P<$ $0,05)$. Estos resultados indican que los factores determinantes de la EVN afectan de forma diferente a los dos sexos.

Las regresiones múltiples robustas más parsimoniosas y sin colinealidad solo utilizaron la MEG, el IPDH, el IAM, el coeficiente de Gini y la tasa migratoria neta como factores de pronóstico de la EVN (cuadro 3). En el caso de las mujeres se utilizó también el término de interacción entre el coeficiente de Gini y el IPDH. Al evitar la colinealidad se logró aislar mejor los efectos de cada variable empleada, aunque para su interpretación se deben tener presentes las correlaciones más fuertes descritas en el cuadro 1.

Los datos del Distrito Federal y de Quintana Roo no se tomaron en cuenta en los modelos de estimación de la MEG, ya que presentaron distancias de Cook $>1$. Por lo tanto, estos resultados no representan la situación en estos estados federativos. Los ponderadores de los 30 estados utilizados en las estimaciones variaron entre $0,77 \mathrm{y}$ 0,99 en la población total (de 0,76 a 0,99 en hombres y de 0,73 a 0,99 en mujeres). Se observó un efecto negativo $(P<0,05)$ de la MEG sobre la EVN 
FIGURA 1. Relaciones entre las medidas de empoderamiento y la esperanza de vida al nacer, según el estado federativo. México, 2003
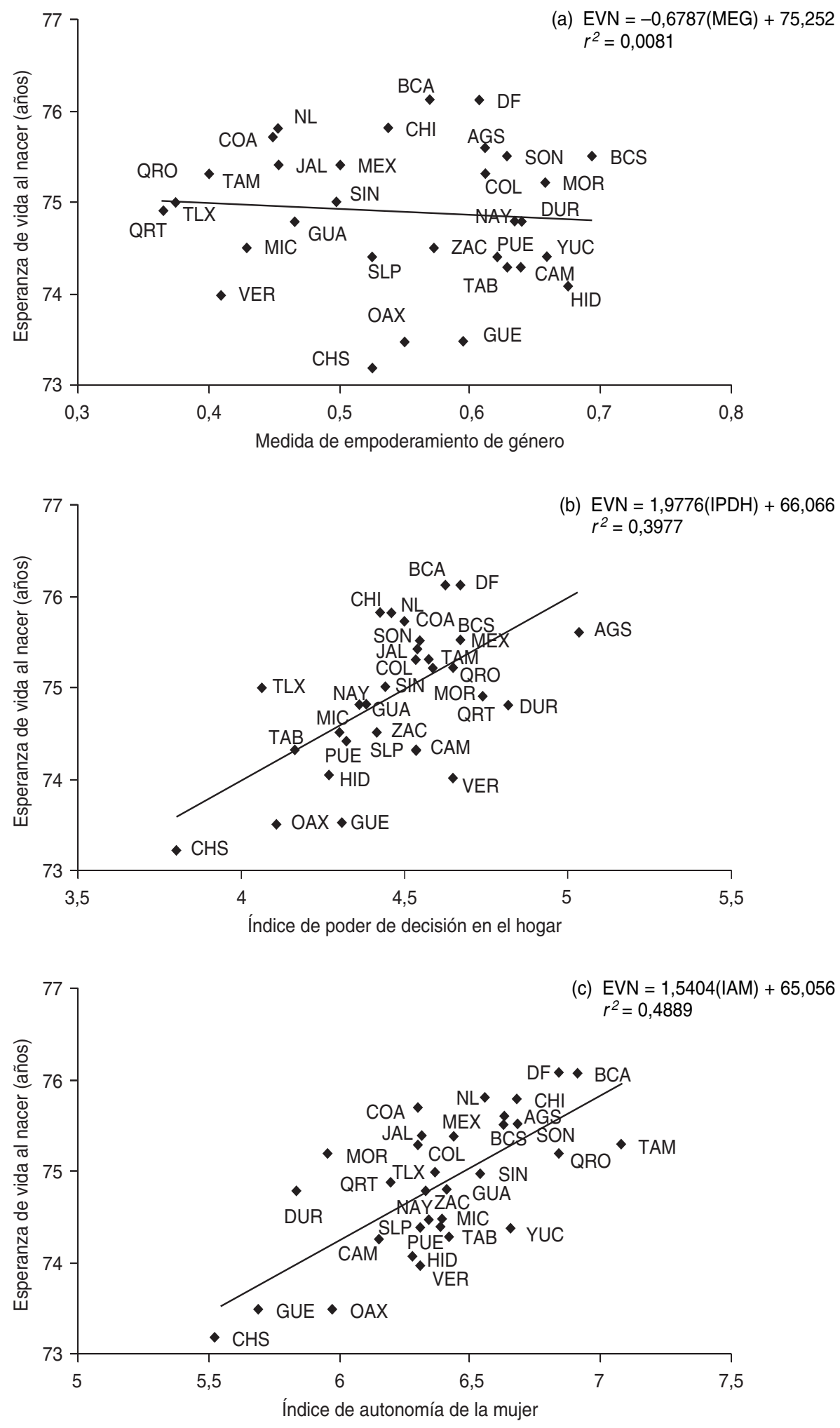

Fuente: Estimado con datos de la Encuesta Nacional sobre la Dinámica de las Relaciones en los Hogares 2003 (ENDIREH) (26).

MEG: Medida de empoderamiento de género; IPDH: índice del poder de decisión de la mujer en el hogar; IAM: índice de la autonomía de la mujer. de las mujeres y total, pero no en la EVN de los hombres. Después de ajustar según el coeficiente de Gini y la tasa migratoria neta, no se observaron efectos significativos del IPDH sobre la EVN. Los modelos con el IAM demostraron un efecto positivo significativo $(P<0,05)$ sobre la EVN total y por sexo y ese efecto fue mayor entre los hombres $(1,78)$ que entre las mujeres $(1,36)$. No se excluyeron estados federativos en ninguno de estos modelos, aunque los ponderadores oscilaron entre 0,53 y 0,99 para la EVN total, entre 0,57 y 0,99 en hombres y entre 0,63 y 0,99 en mujeres.

\section{DISCUSIÓN}

A pesar de que solo se esperaba encontrar efectos positivos del EM sobre la EVN, en esta investigación se hallaron también efectos negativos. La evaluación empírica de datos recientes de los 32 estados federativos mexicanos indica que la MEG mostró una asociación inversa con la EVN en las mujeres, mientras que el IAM tuvo una asociación directa con la EVN total y por sexo. Esto contrasta con los pocos estudios poblacionales similares que exploran los efectos del EM en el nivel de agregación poblacional en Estados Unidos $(14,15)$ y otros que emplearon análisis de niveles múltiples $(16,17)$ y que mostraron un efecto positivo del EM.

Muchos especialistas afirman que la mortalidad infantil es uno de los índices más reveladores del bienestar social y del progreso de un país en materia de salud y que su disminución se traduce en incrementos de la EVN (37). La principal limitación de la EVN como indicador de bienestar social es su estrecha relación inversa con la mortalidad y su relación menos fuerte con la morbilidad y la discapacidad (38). Kawachi et al. encontraron que la mortalidad infantil disminuye significativamente cuando se incrementan la participación política $\left(\beta_{\mathrm{a}}:-0,11\right)$, el empleo y las ganancias $\left(\beta_{\mathrm{a}}:-1,36\right)$, la autonomía económica $\left(\beta_{\mathrm{a}}:-2,17\right)$ y los derechos reproductivos $\left(\beta_{\mathrm{a}}:-0,39\right)$ (14). Los resultados expuestos en el presente artículo indican una relación inversa 
CUADRO 2. Impacto del empoderamiento de la mujer, la desigualdad en el ingreso, el ambiente físico ${ }^{\mathrm{a}}$ y la tasa migratoria neta sobre la esperanza de vida al nacer en los estados federativos mexicanos, estimado mediante modelos de regresión robusta, 2003

\begin{tabular}{|c|c|c|c|c|c|c|c|c|c|}
\hline \multirow[b]{3}{*}{ Variables } & \multicolumn{9}{|c|}{ Esperanza de vida al nacer } \\
\hline & \multicolumn{3}{|c|}{ Total } & \multicolumn{3}{|c|}{ Hombres } & \multicolumn{3}{|c|}{ Mujeres } \\
\hline & $\beta$ & \multicolumn{2}{|c|}{ IC95\% ${ }^{\mathrm{b}}$} & $\beta$ & \multicolumn{2}{|c|}{ IC95\% } & $\beta$ & \multicolumn{2}{|c|}{ IC95\% } \\
\hline Índice de poder de decisión en el hogar (IPDH) & $2,07^{d}$ & 1,07 & 3,07 & $2,30^{d}$ & 1,20 & 3,41 & $1,79^{d}$ & 0,88 & 2,70 \\
\hline Índice de autonomía de la mujer (IAM) & $1,59^{d}$ & 0,95 & 2,23 & $1,78^{\mathrm{d}}$ & 1,06 & 2,51 & $1,36^{\mathrm{d}}$ & 0,79 & 1,93 \\
\hline Coeficiente de Gini & $-10,11^{d}$ & $-14,46$ & $-5,76$ & $-11,28^{d}$ & $-16,25$ & $-6,31$ & $-8,89^{d}$ & $-12,76$ & $-5,03$ \\
\hline FC2 & 0,14 & $-0,16$ & 0,44 & 0,14 & $-0,20$ & 0,47 & 0,13 & $-0,13$ & 0,39 \\
\hline FC3 & 0,11 & $-0,18$ & 0,40 & 0,15 & $-0,18$ & 0,47 & 0,08 & $-0,17$ & 0,34 \\
\hline FC4 & 0,10 & $-0,19$ & 0,40 & 0,12 & $-0,21$ & 0,45 & 0,10 & $-0,16$ & 0,36 \\
\hline Tasa migratoria neta & $0,71^{d}$ & 0,41 & 1,00 & $0,74^{\mathrm{d}}$ & 0,40 & 1,08 & $0,66^{\mathrm{d}}$ & 0,42 & 0,90 \\
\hline Interacción MEG-coeficiente de Gini & $-33,89$ & $-82,01$ & 14,24 & $-41,67^{e}$ & $-95,91$ & 12,57 & $-29,81$ & $-73,12$ & 13,49 \\
\hline Interacción coeficiente de Gini-IPDH & 5,54 & $-6,27$ & 17,34 & 5,49 & $-7,37$ & 18,35 & $12,81^{\mathrm{e}}$ & $-2,74$ & 28,36 \\
\hline
\end{tabular}

Fuente: Estimados a partir de los datos de la Encuesta Nacional sobre la Dinámica de las Relaciones en los Hogares 2003 (ENDIREH) (26).

a FC1: vulnerabilidad/susceptibilidad poblacional y biodiversidad; FC2: urbanización, industrialización y sustentabilidad ambiental; FC3: resiliencia ecológica; FC4: ambientes libres de plagas forestales. Todos ellos, factores del ambiente físico asociados con la esperanza de vida al nacer.

b IC95\%: intervalo de confianza de 95\%.

${ }^{c}$ MEG: Medida de empoderamiento de género, propuesta por el Programa de las Naciones Unidas para el Desarrollo.

d Significación estadística $P<0,05$

e Significación estadística $P<0,15$

CUADRO 3. Impacto del empoderamiento de la mujer ajustado en función de la desigualdad en el ingreso y la tasa migratoria neta sobre la esperanza de vida al nacer en los estados federativos mexicanos, estimado mediante modelos de regresión robusta, 2003

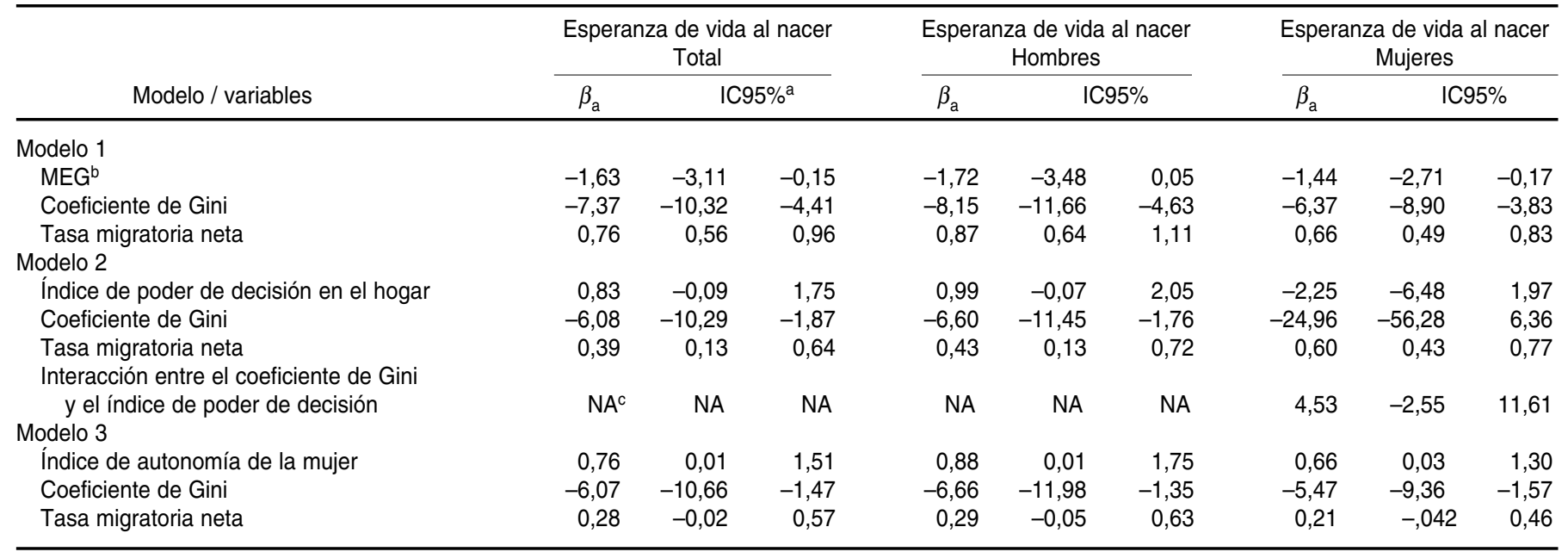

Fuente: Estimado con datos de la Encuesta Nacional sobre la Dinámica de las Relaciones en los Hogares 2003 (ENDIREH) (26).

a IC95\%: intervalo de confianza de 95\%.

b MEG: Medida de empoderamiento de género, propuesta por el Programa de las Naciones Unidas para el Desarrollo.

c NA: no se aplica.

entre la MEG y el EM en México ( $\beta$ : $-1,63)$ y una asociación directa entre el IAM y el EM $\left(\beta_{\mathrm{a}}: 0,76\right)$.

La falta de coherencia en los resultados cuando se usa la MEG como indicador del EM puede deberse al tipo de evento de salud seleccionado, a problemas en la medición del EM o a las particularidades de la sociedad mexicana. En primer lugar, es posible que la EVN no sea un evento totalmente comparable entre los dos sexos, ya que la mortalidad infantil es diferente para hombres y mujeres, tal como demostraron recientemente Tsuchiya y Williams (39). Este efecto diferencial entre los sexos no afectó a nuestro estudio, ya que los análisis se realizaron por 
CUADRO 4. Correlaciones de Spearman entre la medida de empoderamiento propuestas por el Programa de las Naciones Unidas para el Desarrollo (MEG) y las propuestas por el Foro Económico Mundial

\begin{tabular}{|c|c|c|c|c|c|c|c|}
\hline Medidas $^{a}$ & MEG & MEM & $\begin{array}{l}\text { Participación } \\
\text { económica }\end{array}$ & $\begin{array}{l}\text { Oportunidad } \\
\text { económica }\end{array}$ & $\begin{array}{l}\text { Empoderamiento } \\
\text { político }\end{array}$ & $\begin{array}{l}\text { Logros } \\
\text { educativos }\end{array}$ & $\begin{array}{l}\text { Salud y } \\
\text { bienestar }\end{array}$ \\
\hline IDG & $\begin{array}{c}0,79^{b} \\
(0,69 ; 0,86)\end{array}$ & $\begin{array}{c}0,73^{b} \\
(0,58 ; 0,83)\end{array}$ & $\begin{array}{c}-0,24 \\
(-0,47 ; 0,02)\end{array}$ & $\begin{array}{c}-0,30^{\mathrm{b}} \\
(-0,52 ;-0,05)\end{array}$ & $\begin{array}{c}-0,55^{\mathrm{b}} \\
(-0,71 ;-0,35)\end{array}$ & $\begin{array}{c}-0,61^{\mathrm{b}} \\
(-0,75 ;-0,42)\end{array}$ & $\begin{array}{c}-0,67^{\mathrm{b}} \\
(-0,79 ;-0,50)\end{array}$ \\
\hline MEG & 1 & $\begin{array}{c}0,83^{b} \\
(0,71 ; 0,90)\end{array}$ & $\begin{array}{c}-0,32^{\mathrm{b}} \\
(-0,56 ;-0,05)\end{array}$ & $\begin{array}{c}-0,40^{\mathrm{b}} \\
(-0,61 ;-0,13)\end{array}$ & $\begin{array}{c}-0,79^{\mathrm{b}} \\
(-0,88 ;-0,66)\end{array}$ & $\begin{array}{c}-0,58^{\mathrm{b}} \\
(-0,74 ;-0,36)\end{array}$ & $\begin{array}{c}-0,67^{\mathrm{b}} \\
(-0,80 ;-0,48)\end{array}$ \\
\hline MEM & & 1 & $\begin{array}{c}-0,59^{\mathrm{b}} \\
(-0,74 ;-0,40)\end{array}$ & $\begin{array}{c}-0,55^{\mathrm{b}} \\
(-0,71 ;-0,34)\end{array}$ & $\begin{array}{c}-0,84^{\mathrm{b}} \\
(-0,90 ;-0,74)\end{array}$ & $\begin{array}{c}-0,74^{\mathrm{b}} \\
(-0,84 ;-0,59)\end{array}$ & $\begin{array}{c}-0,46^{\mathrm{b}} \\
(-0,64 ;-0,23)\end{array}$ \\
\hline Oportunidad económica & & & & 1 & $\begin{array}{c}0,30^{\mathrm{b}} \\
(0,05 ; 0,52)\end{array}$ & $\begin{array}{c}0,35^{\mathrm{b}} \\
(0,10 ; 0,56)\end{array}$ & $\begin{array}{c}0,04 \\
(-0,22 ; 0,30)\end{array}$ \\
\hline Empoderamiento político & & & & & 1 & $\begin{array}{c}0,55^{\mathrm{b}} \\
(0,34 ; 0,71)\end{array}$ & $\begin{array}{c}0,38^{b} \\
(0,14 ; 0,58)\end{array}$ \\
\hline Logros educativos & & & & & & 1 & $\begin{array}{c}0,23 \\
(-0,04 ; 0,46)\end{array}$ \\
\hline
\end{tabular}

Fuente: Con datos disponibles en las referencias 41 y 42.

a IDG: Índice de desarrollo relacionado con género, propuesto por el Programa de las Naciones Unidas para el Desarrollo; MEG: medida de empoderamiento de género, propuesta por el Programa de las Naciones Unidas para el Desarrollo; MEM: Medida de empoderamiento de la mujer, propuesto por el Foro Económico Mundial.

b Significación estadística $P<0,05$.

separado para hombres y mujeres. La segunda explicación de las contradicciones encontradas se relaciona con problemas de validez del indicador, es decir, la MEG no capta todas las aristas del EM como fenómeno social. Las críticas realizadas a la MEG como indicador se pueden resumir en lo siguiente: a) no toma en cuenta elementos importantes del EM, como la división del trabajo doméstico y el trabajo sin salario (5); b) no considera las diferencias dentro de las naciones, que pueden ser factores determinantes de gran importancia del nivel de EM (por ejemplo, las diferencias en el trabajo asalariado en la agricultura en comparación con el trabajo en labores no agrícolas); c) la aversión $(\epsilon)$ a la desigualdad ${ }^{4}$ usada en la construcción del indicador no es sensible a las diferencias culturales y a las normas sociales (24), en este caso las relacionadas con la amplia diversidad de la población mexicana; d) no utiliza para su construcción indicadores relacionados con la economía y la política, estrechamente asociados con

\footnotetext{
4 La aversión es una constante usada durante la construcción del indicador MEG para ponderar las diferencias entre los hombres y las mujeres.
}

esas mismas dimensiones del EM; y e) se basa en la premisa de que los países con gran desarrollo económico se comportan igual que los países de menor nivel de desarrollo (40). Si bien estas carencias o debilidades de la MEG pueden explicar las contradicciones en los resultados obtenidos, también es importante reconocer que este indicador ha mostrado su utilidad para hacer comparaciones internacionales. No obstante, la MEG no debe aplicarse por igual a otros niveles de agregación, como los estados federativos o los municipios, ya que en ellos se presenta una menor variabilidad.

La disponibilidad de mediciones recientes del EM hechas por el Foro Económico Mundial (41), en las que se utilizó como dimensiones la participación económica, la oportunidad económica, el empoderamiento político, los logros académicos y su relación con la salud y el bienestar, permite comparar esas mediciones del EM con las medidas propuestas por el PNUD (la MEG y el índice de desarrollo relacionado con género). A pesar de la alta correlación observada $(\rho>0,73 ; P<0,05)$ entre esos indicadores y el puntaje total del indicador propuesto por el Foro Económico Mundial para el año
2004 (42) (cuadro 4), las dimensiones utilizadas por esos indicadores tienen grandes diferencias entre sí. Es posible que su aplicación en análisis similares al aquí realizado pueda contribuir a profundizar más en los efectos - tanto positivos como negativos- del EM sobre la EVN. Se debe tener en cuenta que el EM es un proceso amplio que abarca aspectos muy disímiles - como la libertad de movilización, el acceso a recursos financieros y no financieros, la autonomía en la toma de decisiones, las actitudes de género, la libertad frente al miedo y la coerción y la igualdad en sus relaciones con su compañero, otros miembros de la familia y otras mujeres (43) - que no toma en cuenta el indicador de EM de nivel poblacional aquí empleado.

La última posible explicación de los resultados expuestos en este artículo es que realmente ocurran efectos negativos asociados con el EM que no sean producto de un artefacto metodológico. Esta opción no resulta completamente ilógica, ya que existen pruebas de que algunos indicadores de EM en México pueden tener ciertos efectos inmediatos negativos. Por ejemplo, se ha demostrado que las mujeres con un mayor nivel de autonomía presentan 
un mayor riesgo de ser víctimas de violencia emocional por parte de su pareja (44). Por otra parte, dado que la MEG hace mayor hincapié en su construcción en las dimensiones política y económica, es posible que el EM relacionado con estas dimensiones tenga un efecto negativo sobre la EVN. Por ejemplo, en los países en vías de desarrollo - como México- el estatus económico de la mujer es un importante factor determinante de EVN en las mujeres, que se manifestará en dependencia del nivel de desarrollo económico. Sin embargo, esta relación es compleja y sus efectos positivos se asocian más con la ocupación en los sectores industrial o de servicios que con los trabajos en el sector agrícola, ya que estos están asociados con una menor EVN (13).

Los resultados aquí descritos tienen algunas limitaciones importantes que se deben tomar en cuenta. El diseño transversal de este trabajo no permite establecer relaciones causales (18); incluso, es posible que exista una causalidad inversa. Para minimizar este efecto se utilizó la EVN como evento de salud, ya que se ajusta mejor a estos diseños por corresponder la ventana de exposición a un período mínimo de latencia para la aparición del evento y este depende en gran medida de la mortalidad de menores de un año (22). Si bien algunas variables independientes se calcularon con datos del año 2000 y la EVN y otras variables con datos de 2003, se buscó respetar la precedencia de las exposiciones a los eventos medidos. Los datos presentados tampoco permiten hacer inferencias a nivel individual y solo pueden entenderse desde el punto de vista poblacional. Intentar hacer inferencias a los individuos puede ocasionar una falacia ecológica (45), ya que los factores determinantes poblacionales de la incidencia de un evento de salud no necesariamente son los mismos que determinan su ocurrencia en individuos (46). Finalmente, la alta colinealidad observada entre las variables hace difícil aislar el efecto de cada unpa de estas, lo cual sin duda influyó en la significación estadística (18).

En conclusión, el uso de la MEG como aproximación al EM - pese a ser uno de los indicadores más difundidos y reconocidos en el mundo- no puso de manifiesto efectos positivos del EM sobre la EVN en México. Esto indica que la MEG calculada para los estados federativos mexicanos no es un indicador adecuado del EM y que su uso debe limitarse a análisis con niveles mayores de agregación. En este sen- tido, se deben revisar los elementos que integran la MEG y su pertinencia en diversos contextos. El IAM mostró una mayor asociación con la EVN y su efecto fue de mayor magnitud entre los hombres. Este indicador permitió medir la EM en México y se recomienda su empleo, mientras no se tenga otro que permita captar más eficazmente todos los elementos que inciden en el EM.

Los resultados contradictorios en la relación de la EVN con los indicadores de EM pueden obedecer a la diferente naturaleza de los indicadores (unidimensional o multidimensional), al diferente nivel en que originalmente se midieron (individual o poblacional) o incluso a los diferentes elementos del EM que abarcan. Para poder avanzar en la comprensión de las relaciones entre el EM y la EVN es necesario realizar nuevos estudios, tanto con indicadores individuales como poblacionales - unidimensionales y multidimensionales en ambos casos- y diseñar otros indicadores que reflejen todas las dimensiones del EM. Asimismo, se recomienda realizar estudios de múltiples niveles de agregación que permitan poner de manifiesto el efecto contextual del EM sobre la salud individual, no explorado en este estudio.

\section{REFERENCIAS}

1. Lewis O. Life in a Mexican village: Tepoztlán revisited. Chicago: University of Illinois Press; 1951.

2. Oropesa RS. Development and marital power in Mexico. Soc Forces. 1997;75:1291-317.

3. Casique I. Power, autonomy and division of labor in Mexican dual-earner families. Lanham, MD: University Press of America; 2001.

4. Batliwala S. The meaning of women's empowerment: New concepts from action. En: Sen G, Germain A, Chen LC, eds. Population policies reconsidered. Health, empowerment and rights. Cambridge: Harvard University Press; 1994. Pp. 127-38.

5. Hobcraft J. The consequences of female empowerment for child well-being: a review of concepts, issues, and evidence in a post-Cairo context. En: Presser HB, Sen G, eds. Women's empowerment and demographic processes. Moving beyond Cairo. New York: Oxford University Press; 2000: 159-85.
6. Hobcraft JN. Women's education, child welfare and child survival: a review of the evidence. Health Trans Rev. 1993;3:159-75.

7. Reed BA, Habicht JP, Niameogo C. The effects of maternal education on child nutritional status depend on socio-environmental conditions. Int J Epidemiol. 1996;25:585-92.

8. Kar SB, Pascual CA, Chickering KL. Empowerment of women for health promotion: a meta-analysis. Soc Sci Med. 1999;49:1431-60.

9. Hemstrom $\mathrm{O}$. Health inequalities by wage income in Sweden: the role of work environment. Soc Sci Med. 2005;61:637-47.

10. Artazcoz L, Borrell C, Benach J, Cortés I, Rohlfs I. Women, family demands and health: the importance of employment status and socio-economic position. Soc Sci Med. 2004; 59:263-74

11. Nath DC, Land KC, Goswami G. Effects of the status of women on the first-birth interval in Indian urban society. J Biosoc Sci. 1999;31: 55-69.
12. Hunter SS. Levels of health development: a new tool for comparative research and policy formulation. Soc Sci Med. 1990;31:433-44.

13. Williamson JB, Boehmer U. Female life expectancy, gender stratification, health status, and level of economic development: a crossnational study of less developed countries. Soc Sci Med. 1997;45:305-17.

14. Kawachi I, Kennedy BP, Gupta V, ProthrowStith D. Women's status and the health of women and men: a view from the States. Soc Sci Med. 1999;48:21-32.

15. Yllo K. Sexual equality and violence against wives in American states. J Comp Fam Stud. 1983;14:67-86.

16. Jun HJ, Subramanian SV, Gortmaker S, Kawachi I. A multilevel analysis of women's status and self-rated health in the United States. J Am Med Women Assoc. 2004;59: 172-80.

17. Chen YY, Subramanian SV, Acevedo-García D, Kawachi I. Women's status and depressive 
symptoms: a multilevel analysis. Soc Sci Med. 2005;60:49-60.

18. Morgenstern H. Ecologic studies in epidemiology: concepts, principles, and methods. Annu Rev Public Health. 1995;16:61-81.

19. Instituto Nacional de Estadística, Geografía e Informática. Esperanza de vida por entidad federativa según sexo, 2005. México, D.F.: INEGI; 2005. Hallado en: http://www. inegi.gob.mx/est/contenidos/espanol/ rutinas $/$ ept.asp? $t=$ mpob56\&c $=3233$. Acceso el 15 de febrero de 2005.

20. McCann JC. A technique for estimating life expectancy with crude vital rates. Demography. 1976;13:259-72.

21. Arriaga EE. Measuring and explaining the change in life expectancies. Demography. 1984;21:83-96.

22. Coburn D. Beyond the income inequality hypothesis: class, neo-liberalism, and health inequalities. Soc Sci Med. 2004;58:41-56.

23. United Nations Development Programme. Human development report. New York: Oxford University Press; 1997.

24. Gormerly PJ. The human development index in 1994: impact of income on country rank. J Econ Soc Meas. 1995;21:253-67.

25. Ibarrarán ME, Robles $C$. Inequidad de género en desarrollo humano: el caso de México. En: López-Calva LF, Székely M, eds. La medición del desarrollo humano en México. México, D.F.: Fondo de la Cultura Económica; 2005.

26. Instituto Nacional de Estadística, Geografía e Informática. Encuesta Nacional sobre la Dinámica de las Relaciones en los Hogares 2003 (ENDIREH). Aguascalientes, México: INEGI; 2004. Pp. 1-147.

27. Casique I. Índices de empoderamiento femenino y su relación con la violencia de género.
En: Instituto Nacional de las Mujeres. Violencia de género en las parejas. Resultados de la Encuesta Nacional sobre la Dinámica de las Relaciones en los Hogares 2003. México, D.F.: Inmujeres; 2004. Pp. 75-107.

28. Mulaik SA. A brief history of the philosophical foundations of exploratory factor analysis. Multiv Behav Res. 1987;22:267-305.

29. Cronbach LJ. Coefficient alpha and the internal structure of tests. Psychometrika. 1951;16: 297-334.

30. Kawachi I, Kennedy BP, Glass R. Social capital and self-rated health: a contextual analysis. Am J Public Health. 1999;89:1187-93.

31. Idrovo AJ. Desigualdad en el ingreso, corrupción y esperanza de vida al nacer en México. Rev Salud Publica (Bogotá). 2005;7:121-9.

32. Tuirán-Gutiérrez R. La distribución del ingreso monetario en México. Este país. Tendencias y opiniones. 2003;142:1-5.

33. Milanovic B. A simple way to calculate the Gini coefficient, and some implications. Econ Lett. 1997;546:45-9.

34. Estados Unidos Mexicanos, Secretaría del Medio Ambiente y Recursos Naturales. Compendio de estadísticas ambientales. México, D.F.: SEMARNAT; 2005. Disponible en http:// www.semarnat.gob.mx/dgeia/informe_04/ sitio_wf/Index.htm. Acceso el 17 de mayo de 2006.

35. Huber PJ. Regression. En: Robust statistics. New York: John Wiley \& Sons; 1981. Pp. 153-98.

36. Morgenstern H. Ecologic studies in epidemiology: concepts, principles, and methods. Annu Rev Public Health. 1995;16:61-81.

37. Vaupel JM. Life expectancy at current rates vs current conditions. Demog Res. 2002;7(Article 8). Hallado en: http://www.demographic- research.org/volumes/vol7/8/7-8.pdf. Acceso el 21 de julio de 2006.

38. Gold MR, Stevenson D, Fryback DG. HALYS and QALYS and DALYS, oh my: similarities and differences in summary measures of population health. Annu Rev Public Health. 2002; 23:115-34.

39. Tsuchiya A, Williams AA. "Fair innings" between the sexes: are men being treated inequitably? Soc Sci Med. 2005;60:277-86.

40. Pilarisetti JR, McGillivray M. Human development and gender empowerment: methodological and measurement issues. Dev Pol Rev. 1998;16:197-203.

41. World Economic Forum. Women's empowerment: measuring the global gender gap. Geneve: World Economic Forum; 2005.

42. United Nations Development Programme. Human Development Report 2004. Cultural liberty in today's diverse world. New York: UNDP; 2004.

43. Federici N, Mason KO, Sogner S. Introduction. En: Women's position and demographic change. Oxford Clarendon Press; 1993. Pp. 1-15.

44. Casique I. ¿Cuándo puedo decir no? Empoderamiento femenino y sexo no deseado en México. Estud Demogr Urb. 2006;21:49-81.

45. Robinson WS. Ecological correlations and the behavior of individuals. Am Sociol Rev. 1950; 15:351-7.

46. Rose G. Sick individuals and sick populations. Int J Epidemiol. 1985;14:32-8.

Manuscrito recibido el 19 de septiembre de 2005. Aceptado para publicación, tras revisión, el 1 de junio de 2006.
ABSTRACT

Women's empowerment and life expectancy at birth in Mexico

Key words Life expectancy, health status indicators, women's rights, Mexico.
Objectives. To assess the effect of women's empowerment (WE) on life expectancy at birth (LEB) in the federative states of Mexico and to compare the results of measuring WE with various compound indicators that reflect, to a greater or lesser degree, an individual or population focus.

Methods. This was an ecological study conducted in Mexico's 32 federative states. We estimated the correlations between overall and sex-specific LEB on the one hand, and a measure of gender empowerment (MGE), the index of women's ability to make decisions within the household (WADH), the index of women's autonomy (IWA), income inequality, certain aspects of the physical environment, the proportion of the population who spoke an indigenous language, and the net migratory rate on the other. By using robust regressions, we studied the effect on LEB of MGE, IWA, and WADH, after mutually adjusting for other independent variables.

Results. A very strong inverse correlation (-0.93) was found between overall LEB and factors of the physical environment linked to population vulnerability and biodiversity. Significant direct and inverse correlations were also found between LEB on the one hand and WADH, IWA, net migratory rate, the percentage of the population that spoke an indigenous language, and the Gini coefficient on the other. Multiple robust regressions showed inverse associations between MGE and LEB in women $(\beta$ : -1.44 ; $95 \%$ confidence interval [ $95 \% \mathrm{CI}]:-2.71$ to -0.17$)$. WAI was positively associated with LEB in men ( $\beta$ : $0.88 ; 95 \%$ CI: 0.01 to 1.75$)$ and women $(\beta: 0.66 ; 95 \%$ CI: 0.03 to 1.30$)$. Conclusion. The use of MGE as a surrogate for WE failed to reveal a positive effect of WE on LEB in Mexico. It is necessary to review the components that make up MGE and the relevance of using such a measure in different contexts. WAI showed a greater association with LEB and its effect was greater among men. This indicator made it possible to measure WE in Mexico and its use is recommended, as long as there are no other indicators available for capturing more effectively all the components that affect WE. 